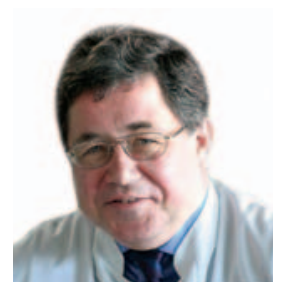

Prof. Dr. Hans F. Merk

hans.merk@post.rwth-aachen.de

\title{
Dermatologie - kompakt und praxisnah vermittelt
}

Das Konzept einer kompakten Wissensvermittlung für die Dermatologie erfreut sich in Deutschland wachsender Beliebtheit. Die Deutsche Dermatologische Gesellschaft verfolgt mit ihrem Kongress «DDG KOMPAKT - Fortbildung für die deutschsprachige Dermatologie» eine ähnliche Idee, wie sie auch dem «Stapellauf» von Karger Kompass Dermatologie zugrunde lag. Mit der zweiten Ausgabe von Karger Kompass Dermatologie wird dieser Weg unter der bewährten Koordination von Herrn Professor Lademann nun fortgesetzt.

In den beiden hier in deutscher Übersetzung erscheinenden Übersichtsarbeiten zu Beginn des Heftes werden zentrale aktuelle Fragestellungen der Dermatologie thematisiert. So werden in der ersten Übersichtsarbeit aus der Arbeitsgruppe von Lajos Kemény Zusammenhänge zwischen Entzündungsreaktionen der Haut und der Leber mit Fokus Psoriasis dargestellt. Die darauf folgende Übersichtsarbeit aus der Arbeitsgruppe von Vera Rogiers setzt sich mit aktuellen Entwicklungen bei den In-vitro-Ersatzmethoden für Tierexperimente speziell im Bereich der Kosmetika auseinander. Dabei steht vor allem die Bedeutung von HistonDeacetylase-Inhibitoren für die Etablierung von In-vitro-Hautmodellen im Vordergrund - eine Thematik, zu der gerade die Arbeitsgruppe von Frau Rogiers wesentliche Ergebnisse beigetragen hat. Nach diesem interessanten Auftakt werden im Rahmen des Wissenstransfers zahlreiche weitere Entwicklungen im Bereich Dermatologie in Bezug auf ihre Praxisrelevanz kommentiert, z.B. die Prävention der besonders unangenehmen palmar-plantaren Erythrodysästhesien bei der Chemotherapie, die Problematik von Formaldehyd in Körperpflegeprodukten, endokrinologische Aspekte bei Akne sowie die Hautalterung. Darüber hinaus beschäftigen sich Beiträge mit der optimalen Durchführung eines PrickTests bei Kindern, der PolyhexanidAnwendung und der Klassifizierung der Acrodermatitis continua Hallopeau.

Den Abschluss bilden zwei Berichte zu Erfahrungen aus der Praxis, die sich zum einen am Beispiel der Skabies-Infektion mit der diagnostischen Anwendung der optischen Kohärenztomographie beschäftigen und zum anderen eine besonders bemerkenswerte Melanom-Kasuistik vorstellen.

Zu dieser wiederum außerordentlich interessant zusammengestellten Ausgabe von Karger Kompass Dermatologie kann man dem Verlag und den Kollegen des Beirates in jeder Hinsicht gratulieren.

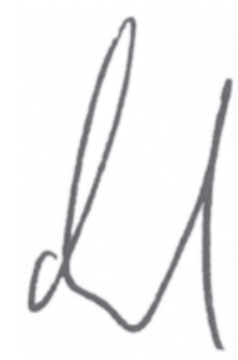

\section{KARGER}

Fax +497614520714

information@karger.com www.karger.com
() 2014 S. Karger GmbH, Freiburg

2296-5424/14/0021-0005\$39.50/0

Accessible online at:

www.karger.com/kkd
Univ.-Prof. Dr. med. Hans F. Merk Universitäts-Hautklinik

RWTH Aachen

Pauwelsstraße 30, 52074 Aachen, Deutschland 